\title{
Value-Based HIV Care: A Method to Improve Quality of HIV Care.
}

Guido van den berk ( $\nabla$ g.e.l.vandenberk@olvg.nl )

OLVG https://orcid.org/0000-0003-4417-7601

\section{Daoud Ait Moha}

OLVG

Janneke Stalenhoef

OLVG

Marie-Jose Kleene

OLVG

Narda van der Meche

OLVG

Ineke Schouten

OLVG

Samyra Keus

OLVG

Kees Brinkman

OLVG

\section{Methodology}

Keywords: HIV , health outcomes, VBHiC

Posted Date: December 3rd, 2020

DOI: https://doi.org/10.21203/rs.3.rs-117180/v1

License: (c) (i) This work is licensed under a Creative Commons Attribution 4.0 International License.

Read Full License 


\section{Value-Based HIV Care: a method to improve quality of HIV care.}

Guido van den Berk', Daoud Ait Moha1', Janneke Stalenhoef', Marie-Jose Kleene', Narda van der Meche ${ }^{1}$, Ineke Schouten ${ }^{1}$, Samyra Keus ${ }^{2}$, Kees Brinkman ${ }^{1}$ on behalf of the OLVG HIV care team

1OLVG, Department of Internal Medicine and Infectious Diseases, Amsterdam

${ }^{2}$ OLVG, Department of Quality and Improvement, Amsterdam 


\section{Background}

In 2014, the Joint United Nations Programme on HIV and Aids (UNAIDS) set the "9090-90 targets". The goal was to diagnose $90 \%$ of all people living with HIV; treat $90 \%$ of those diagnosed with HIV with antiretroviral therapy and achieve viral suppression in $90 \%$ of those treated ${ }^{1,2}$.These targets were reached in the Netherlands in $2017^{3}$. A "fourth 90" was proposed in 2016 to ensure that $90 \%$ of people with a suppressed viral load have good health-related quality of care (HrQoL) ${ }^{4}$. In 2014, we started exploring new health goals that went beyond viral suppression and defined parameters to improve both quality of HIV care and quality of life for our patients at OLVG Hospital in Amsterdam, the largest HIV center in the Netherlands. To do this, we applied the value-based health care concept introduced by Michael Porter ${ }^{5}$. In his concept, which had not previously been applied to HIV care, Porter describes six steps to improve health value for patients by improving health outcomes and reducing costs. In this paper, we describe how we applied these six steps and used value-based HIV care (VBHiC) to create an HIV care structure that facilitates continuous monitoring and improvement of relevant quality and cost indicators. 


\section{Methods}

OLVG Hospital, a teaching hospital in Amsterdam's city center with 600 beds, cares for more than 500,000 patients per year, including approximately 3500 people living with HIV (PLHIV). We applied the six steps described by Porter to create a high value HIV care delivery system, as described below. We collected data on health outcome indicators and process indicators retrospectively from electronic patient files for the years 2005 through 2015, with prospective data collection starting in 2015.

\section{Step 1) Organizing into integrated practice units / describing the HIV care path}

The HIV care path starts when the patient is referred to our HIV center. At each point in the care path, we described all necessary diagnostic and therapeutic steps in compliance with our formal standards and national guidelines ${ }^{6}$ and developed clear descriptions of the roles played by the various actors in the care path, such as the patient, the nurse and the doctor's assistant. We labelled each visit in the care path with a specific code and attached a set of specific activities (orders) to each code. These orders represent items such as laboratory tests and quality of life questionnaires, as well as items such as follow up visits. Where necessary, additional tests and visits can be manually added to the care path. Diagnostic tests and therapeutic activities relating to comorbidities were registered outside of the HIV care path. We defined two main HIV care paths: one for the first year of care and one for subsequent years. We also developed parallel care paths for specific, frequently occurring comorbidities such as hepatitis $\mathrm{C}$ infections, as well as for mental health problems such as anxiety and depression.

\section{Step 2) Defining an HIV outcome indicator set}

The international consortium for health outcome measurement (ICHOM) issued a number of health outcome standard sets designed to transform health care systems worldwide based on standardised measurement and reporting of patient outcomes ${ }^{7}$. These standard sets did not include a standard set for HIV health outcomes, however, so we decided to develop one ourselves. We began by searching the literature for terms such as HIV, monitoring, outcome, quality of care, morbidity, comorbidity, mortality, cause of death and admissions. We needed a set of outcomes that covered the full cycle of care for the patient. According to Porter, the outcomes 
that matter to patients for a particular medical condition fall into three tiers: Tier 1 is "Health status achieved or retained", Tier 2 is "Process of recovery" and Tier 3 is "Sustainability of health". We set up three panels of our own patients to perform indepth reviews of potential indicators in a focus group setting. In a working group with HIV health care professionals, a psychologist and a psychiatrist, we identified various quality of life domains based on evaluations of patient referrals for psychological problems over a ten-year period. We also assessed the potential indicators against the results of a quality of life and needs survey conducted by the Dutch HIV patient organization (HVN) of a representative sample of 468 PLHIV in the Netherlands ${ }^{8}$. Ultimately, we selected the 9 outcome indicators with the highest prevalence in the population, the highest impact on the patient and the highest potential to be influenced by the patient and/or caregiver, or a combination of these factors. We analyzed the selected indicators retroactively against the data culled from patient files for the years 2005 through 2015 and prospectively for subsequent years. The indicators quality of life and cardiovascular risk, however, form exceptions to the strategy above. Routine measurement of quality of life began in January 2015 using patient related outcome measures (PROMs), while routine measurement of cardiovascular risk factors, including smoking status, occurs and is recorded in the electronic patient file during every visit.

\section{Step 3: Building an enabling information technology platform}

The HIV care path, with all of its steps, the related codes and the underlying orders, was integrated into our electronic patient file system $\mathrm{EPIC}^{\circledR 9}$ in this step, which resulted in a detailed overview of our care process and the ability to extract discrete data at patient level, process level and population level, thus facilitating assessment of the HIV care that we offer our patients.

\section{Step 4: Integrating care delivery across separate facilities}

In this step, we described and formalized collaboration between the various health care specialisms and caregivers involved in HIV care, including specialists in the areas of pharmacology, medical microbiology, dermatology and psychiatry, as well as technicians in the hospital laboratory and doctors in general medical practices outside of the hospital. 


\section{Step 5: Moving to bundled payments for care cycles}

In this step, we studied the selected indicators in close consultation with a key national health insurer and used the results to develop a new bundled payment contract to cover patient care. The contract is quality-driven and provides for a predetermined payment per patient per year.

\section{Step 6: Expanding services across geographic boundaries.}

This step, which is ongoing, involves the development of formalized methods, guidelines, processes and best practices, based on measurable and verifiable benchmarks and showcased in the application of Value-Based HIV Care in our HIV center at OLVG Hospital, in preparation for a roll out to other HIV centers throughout the Netherlands and elsewhere. 


\section{Results}

\section{Step 1: Organizing into integrated practice units / describing the HIV care path}

Table 1 is a schematic overview of key moments in the patients care path. After referral, an HIV nurse will see the patient within 3 working days for an intake with a duration of one hour (appointment code "0.0"). In cases involving urgent medical problems, the patient will also see an HIV specialist at the same moment. Then, blood and urine analysis are performed, as well as a chest X-ray, and the patient is asked to complete his or her first PROM, which consists of 62 questions. Where possible, the patient is started on combination antiretroviral therapy (cART) during this first intake. When cART is initiated, the patient is given detailed information on the prescribed therapy by an HIV nurse. The HIV nurse, who is assigned to the patient and acts as the patient's contact person for any questions relating to HIV and the prescribed therapy, maintains regular contact with the patient after the therapy is initiated (via the telephone and face-to-face appointments in our HIV center). A faceto-face appointment is scheduled for all patients 4 weeks after CART is initiated (special code for appointment 4 weeks after start or switch cART). In cases in which the patient does not immediately see an HIV doctor due to urgent medical problems, an appointment is scheduled for a 30-minute consultation with an HIV doctor within 2 weeks of the initial intake (code "0.1"). During this consultation, the HIV doctor reviews the patient's medical condition, including the results of CART if therapy was prescribed during the intake (code 0.0). In cases in which the patient has not yet been given therapy, CART is initiated during this consultation. Patients are seen again by the HIV doctor three months later (code " 0.4 "; 10 minutes) and subsequently every 4 months until the end of the first year (code " 0.8 " (10 minutes) and code "1.0" (20 minutes)). Code "1.0" marks the end of the patient's first year of care and represents a 20-minute consultation with the HIV doctor. During this consultation, the patient receives the results his or her annual check-up, which includes lipid profile and blood glucose readings and urine analysis results, as well as a discussion of the patient's PROMs. Patients are asked to complete PROMs before every consultation with the HIV doctor in the first year of care.

After the first year, the patient enters the chronic phase of follow-up, with visits every six months (code "1.6" (10 minutes) and code "2.0" (20 minutes)). The second of 
these two visits (code "2.0"), similarly to the code "1.0" visit at the end of the first year of treatment, includes a review of the patient's PROMs and lab results. During the chronic phase, in the absence of comorbidities or other complications, patients are seen alternately by an HIV nurse (1.6 NS (nurse specialist); 20 minutes) and the HIV doctor (2.0 MS (medical specialist). Where this delegation of tasks to the HIV nurse is unfeasible, the patient is seen twice a year by the doctor (1.6 MS and 2.0 MS). In cases involving complications and comorbidities, the patient will be seen more frequently. In these cases, appointment codes that differ from codes used in the HIV care path are used to distinguish the diagnostic procedures and health care activities performed to handle complications and comorbidities from those that fall within the HIV care path.

\section{Step 2: Defining an HIV outcome indicator set}

Our search of the literature 10111213141516171819202122232425262728293031323311 yielded 38 potential outcome indicators (Appendix A). The nine that we selected to form our HIV outcome indicator set are shown in Table 2.

Figures 1 through 4 show the retrospective evaluation of a number of the selected outcome indicators through 2015. Starting in 2005, an increasing number of patients started HIV therapy soon after care entry. By 2015, $96 \%$ of patients had full viral control in the first year of HIV care (indicator 1, figure 1). Mortality rates in the first year of care were very low and were also very stable over time $(<1 \%)$ (indicators 3 and 9; figure 2). Retention in care was chosen as an important parameter for tier 2. 2780 patients were in care on 1 January 2015 and 183 new patients entered care in the course of the year. Since 'lost to follow up' is defined as 'no visit and/or HIV load measurement for more than 365 days', none of the patients who entered care in 2015 could become lost to follow up in 2015 . Of the total of 2780 patients, 118 patients left care. 22 of those patients died, 18 went to another hospital in the Netherlands, 54 moved abroad and 24 were lost to follow up. With $0.86 \%$ of patients lost to follow up (24 of the 2780 patients), the percentage of patients retained in care in 2015 was 99,14\% (indicator 4; figure 3). Persistence of viral control (for 5 years) increased from $65 \%$ of patients with full viral suppression in 2003 to a stable $90 \%$ in more recent years (indicator 5; figure 4). We assessed therapy tolerance (indicator 6) 
based on CART switches due to toxicity and based on the responses to questions in the PROMs regarding quality of life and cardiovascular risk that were collected from 2015 onward.

\section{Step 3: Building an enabling information technology platform}

The electronic patient file system $\mathrm{EPIC}^{\circledR}$ enables us to monitor our HIV care at patient level, process level and population level. Parameters necessary for indicator surveillance are registered using a special HIV form that is built into EPIC ${ }^{\circledR}$ and that also makes discrete data storage possible. All VBHiC parameters are displayed on a single screen so that the patient and caregiver can see how the patient is performing on each of the parameters, as well as what data may be missing for that patient. All VBHiC data and processes are closely monitored by a data analyst for accuracy and compliance. The data from the system are used for internal evaluation and quality improvement purposes. Each month, a working group of doctors and nurses from our team meets to assess one of the outcome indicators. During these working group meetings, the effects of earlier interventions are evaluated and, if necessary, additional interventions are implemented to continuously improve care through a plan-do-check-act (PCA) cycle.

\section{Step 4: Integrating care delivery across separate facilities}

The HIV care path includes optional referrals to other facilities. Optional referrals include referrals for anal and cervical dysplasia screening, for instance, as well as referrals to a psychologist or psychiatrist in cases involving raised anxiety/depression scores (HADS >15). In the future, we plan to integrate the roles of other caregivers in the HIV care path into our VBHiC system. Among the caregivers we plan to integrate into the system are general practitioners, pharmacists, medical microbiologists and dermatologists.

\section{Step 5: Moving to bundled payments for care cycles}

A three-year value-based health care contract with a key national health insurance provider that provides for bundled payments for HIV care went into effect in January 2018. The contract, which was signed in December 2017 and includes quality parameters from our VBHiC program, contains strict definitions for the care that is covered by the HIV care bundle and for the care that is covered via other provisions. 
Two quality indicators were included for the first year, based on the levels of these indicators in 2016: cardiovascular risk evaluation and cascade of care as a composite indicator of mortality, retention in care and therapy effectiveness. Future plans will include additional quality parameters such as HIV mental health/quality of life, which will help to improve projections of HIV-related health care expenditures outside of the hospital. The contract also includes provisions for cost monitoring and follow-up in time. Figure 5 shows a decline in total laboratory costs and outpatient department (OPD) consultation costs despite growth in the patient population.

\section{Step 6: Expanding services across geographic boundaries.}

Our primary goal in developing our VBHiC program was to improve the care that we offer to patients in our HIV center at OLVG Hospital. Our implementation of VBHiC can serve as a showcase of methodologies and best practices for a nationwide roll out of VBHiC. In 2019, our initiative won the Value Based Healthcare Patients Outcome Award. Our program is highlighted as a good practice on the HIV Outcomes Initiatives website ${ }^{34}$. In 2019, the ICHOM Conference honored our VBHiC program with a poster. Since then, HIV centers at other hospitals in the Netherlands have begun to implement PROMs and other outcome indicators from our program in their own HIV care paths. 


\section{Discussion}

Incorporating value-based HIV care into our daily clinical practice forced us to redefine our care process based on outcome parameters with an added value for our patients. We defined our key outcome parameters in close collaboration with our Dutch patient organization to optimize added value. With a detailed HIV care path (step 1), we are able to focus greater attention on important moments in the HIV care path, thus heightening the visibility of activities within the care path and clarifying expectations, for both the HIV caregiver and the patient. Patients receive the support they need to arrive fully prepared for their consultations in the outpatient clinic and the structured approach helps us to incorporate easily missed but essential aspects of care. Discussions with patients about the impact of smoking on their health are now incorporated into the care path, for instance, as is assessment of the patient's mental health. Every member of our HIV care team participates in one or more of our outcome parameter subgroups. Each month, one of these subgroups conducts a presentation for the entire HIV care team that handles the subgroup's specific outcome parameter in detail, with a focus on best practices and possible improvements, creating an efficient plan-do-check-act cycle (PDCA) that ensures that all aspects of our VBHiC process are evaluated for further improvement as the year progresses. The PDCA cycle is supported by a data manager and quality officer who give regular feedback to the HIV care team. Retrospective evaluation of several of the indicators (step 2) has already yielded interesting results. Our scores for viral suppression over a single year and persistent viral suppression over 5 years (indicators 1 and 5) were already consistently high prior to the introduction of our VBHiC program. Given the importance of this parameter, we decided to keep it in the program as a benchmark for other centers in the Netherlands and abroad. This parameter is particularly important for countries that may have difficulties with initiation and/or continuation of antiretroviral treatment due to resource limitations.

Mortality (indicators 3 and 9) was already consistently low in our hospital, leaving what appeared to be little space for further improvement. However, with an ageing population, we expect efforts to keep mortality low via effective management of comorbidities such as cardiovascular disease to be of high value to our patients in the future. 
At a mere $0.86 \%$, patients lost to follow-up was already quite low in our HIV care center. Still, we developed a protocol to sustain our high level of retention in care (indicator 4), which consists of structured telephone calls, emails and letters sent via normal mail, as well as contact moments with the patient's partner, where relevant, and the patient's general practitioner in cases involving patients who do not receive care for a period of 8 consecutive months.

Therapy tolerance (indicator 5) was a relatively difficult parameter to define. Toxicity can be defined objectively in biochemical or radiological terms (kidney function, lipids, bone mineral density), but the term 'switch due to toxicity' is more subjective and dependent on the viewpoints and attitudes of the doctor and the patient over time. We were unable to find a validated questionnaire for scoring medication tolerance or intolerance. In practice, we expect the structural inclusion of therapy tolerance in our questionnaire to increase patient satisfaction levels.

Evaluation of cardiovascular risk (indicator 7) is now an integral part of the annual consultation. By discussing the 10-year cardiovascular risk with patients and focusing on decreasing cardiovascular risk to less than $10 \%$ for patients ${ }^{11}$ we expect use of statins and medication to reduce hypertension to increase in the short term, but we also hope to persuade more patients to stop smoking as time progresses. Hopefully, our efforts in this area will result in a decline in cardiovascular events and a corresponding decrease in overall cardiovascular costs.

Routine measurement of quality of life using PROMs (indicators 2 and 8 ) was the most innovative outcome indicator in our VBHiC approach. From the moment they were introduced, the PROMs turned out to be a good point of departure for structured discussions of physical and mental health with our patients. Most patients appreciate the holistic approach that the PROMs represent. Approximately $60 \%$ of patients returned their questionnaire in the first year. Of course, logistical problems such as language barriers, privacy concerns and incorrect email addresses need to be overcome with any questionnaire, and it takes time to implement and familiarize patients with the process. Patients who are more difficult to reach may even simply refuse to complete PROMs. We recently set up a project to analyze why certain patients fail to complete PROMs and to develop other methods to evaluate quality of life for these patients. 
Our collaboration with our hospital's psychiatry department is an example of integrating care deliveries across separate facilities (step 4). In the past, we lacked objective criteria for referring patients to the outpatient clinic of psychiatry department. In the VBHiC program, we now use a hospital anxiety depression score as a screening tool and indicator for referrals to the psychiatry department. In addition, attending multidisciplinary meetings and dual consultations with our HIV nurses has resulted in more active involvement in HIV care on the part of the hospital's psychologists and psychiatrists.

Effective communication, an efficient organization and a computer-based care path are required to implement and sustain value-based HIV care. Our HIV care process is built into $E P I C^{\circledR}$. Other HIV centers will need to build their own electronic patient file systems. We have a VBHiC working group for operational issues and a steering group to monitor progress and facilitate future planning and embedding within the hospital organization. The indicator working groups report their initiatives and results every 3 months. Our VBHiC approach attracted the attention of one of the Netherlands' largest health insurer and resulted in a 3-year bundled payment contract (step 5) that we expect to benefit both our patients and the insurer. Other HIV centers in the Netherlands have begun to implement VBHiC based on our outcome indicators (step 6). In the future, we will all benefit from the opportunities for benchmarking and learning from best practices that an ever-widening implementation of VBHiC will bring.

Value-based HIV care closed a gap in the HIV care path, creating a structured system for the evaluation of parameters that are valuable to our patients. It offers us objective, measurable quality indicators for HIV care and has helped us to offer a growing number of patients excellent HIV care without significant increases in the number of caregivers and without significant increases in the overall cost of care. 


\section{Declarations:}

\section{Ethics approval and consent to participate}

The ecthical committee of pour hospital ("ACWO OLVG") approved collection of all used data to improve our care and to submit these data for publication

\section{Consent for publication}

The manuscript does not contain any individual person`s data

\section{Availability of data and materials}

Data supporting the results reported in the article are stored in the "data warehouse" of OLVG hospital and can be obtained if requested.

\section{Competing interests}

There are no competing interests for any of the authors

\section{Funding}

No funding was obtained for this manuscript

\section{Authors' contributions}

All authors have contributed substantially to the conception and design of the work and have approved to the submission of the current manuscript. Furthermore, they have agreed to be personally accountable for the authors`own contributions.

\section{Acknowledgements}

We would like to acknowledge all of our patients who are at the centre of the hiv care team. A special thanks to the national Hiv patient organization "HVN"(Hiv vereniging Nederland"). 
1 Hill A, Pozniak A. HIV treatment cascades: How can all countries reach the UNAIDS 90-90-90 target? In: AIDS. Lippincott Williams and Wilkins, 2015: 2523-5.

2 90-90-90: treatment for all | UNAIDS. https://www.unaids.org/en/resources/909090 (accessed July 20, 2020).

3 (No Title). https://www.hivmonitoring.nl/application/files/9315/2749/8846/2017_HIV_Monitoring_Report_u pdated_version.pdf (accessed July 20, 2020).

4 Lazarus J V., Safreed-Harmon K, Barton SE, et al. Beyond viral suppression of HIV - the new quality of life frontier. BMC Med. 2016; 14: 94.

5 Porter ME. What is value in health care? N. Engl. J. Med. 2010; 363: 2477-81.

6 Richtlijnen - Nederlandse Vereniging van HIV Behandelaren. https://nvhb.nl/richtlijnen/ (accessed July 20, 2020).

7 ICHOM | Healthcare Improvement | Patient-Reported Outcomes. https://www.ichom.org/ (accessed July 20, 2020).

8 Positief Geluid, het onderzoeksrapport by Hiv Vereniging - issuu. https://issuu.com/hivverenigingnederland/docs/rapport_positiefgeluid-Ir (accessed July 20, 2020).

9 Epic $\mid$...with the patient at the heart. https://www.epic.com/ (accessed July 20, 2020).

10 Deeks SG, Lewin SR, Havlir D V. The End of AIDS: HIV Infection as a Chronic Disease. DOI:10.1016/S0140-6736(13)61809-7. 
11 GUIDELINES. 2018 http://www.eacsociety.org (accessed May 5, 2019).

12 Erdbeer G, Sabranski M, Sonntag I, et al. Everything fine so far? Physical and mental health in HIV-infected patients with virological success and long-term exposure to antiretroviral therapy. J Int AIDS Soc 2014; 17: 19673.

13 Ford N, Vitoria M, Penazzato M, et al. Causes of hospital admission among people living with HIV worldwide: A systematic review and meta-analysis. Lancet HIV 2015; 2: e438-44.

14 Smith CJ, Ryom L, Weber R, et al. Trends in underlying causes of death in people with HIV from 1999 to 2011 (D:A:D): a multicohort collaboration. Lancet 2014; 384: 241-8.

15 HIV/AIDS in Europe. Moving from death sentence to chronic disease management. 2017; published online March 18.

https://www.euro.who.int/en/publications/abstracts/hivaids-in-europe.-movingfrom-death-sentence-to-chronic-disease-management (accessed July 20, 2020).

16 Collaboration TATC. Causes of Death in HIV-1-Infected Patients Treated with Antiretroviral Therapy, 1996-2006: Collaborative Analysis of 13 HIV Cohort Studies. Clin Infect Dis 2010; 50: 1387-96.

17 Weber R, Ruppik M, Rickenbach M, et al. Decreasing mortality and changing patterns of causes of death in the Swiss HIV Cohort Study. HIV Med 2013; 14: 195-207.

18 Lake JE, Currier JS. Metabolic disease in HIV infection. Lancet Infect. Dis. 2013; 13: 964-75.

19 Hileman CO, Eckard AR, McComsey GA. Bone loss in HIV: A contemporary review. Curr. Opin. Endocrinol. Diabetes Obes. 2015; 22: 446-51.

20 Martin-Iguacel R, Llibre JM, Friis-Moller N. Risk of Cardiovascular Disease in an Aging HIV Population: Where Are We Now? Curr. HIV/AIDS Rep. 2015; 12: 375-87.

21 Saylor D, Dickens AM, Sacktor N, et al. HIV-associated neurocognitive disorder 
- Pathogenesis and prospects for treatment. Nat. Rev. Neurol. 2016; 12: 23448.

22 Premature age-related comorbidities among HIV-infected persons compared with the general population - PubMed.

https://pubmed.ncbi.nlm.nih.gov/21998278/ (accessed July 20, 2020).

23 A special supplement to the UNAIDS report on the global AIDS epidemic 2013 HIV AND AGING. 2013.

24 Van Der Werf MJ, Ködmön C, Zucs P, Hollo V, Amato-Gauci AJ, Pharris A. Tuberculosis and HIV coinfection in Europe: Looking at one reality from two angles. AIDS 2016; 30: 2845-53.

25 Levett TJ, Cresswell F V., Malik MA, Fisher M, Wright J. Systematic Review of Prevalence and Predictors of Frailty in Individuals with Human Immunodeficiency Virus. J Am Geriatr Soc 2016; 64: 1006-14.

26 Edelman EJ, Tetrault JM, Fiellin DA. Substance use in older HIV-infected patients. Curr. Opin. HIV AIDS. 2014; 9: 317-24.

27 Nideröst S, Imhof C. Aging With HIV in the Era of Antiretroviral Treatment. Gerontol Geriatr Med 2016; 2: 233372141663630.

28 Degroote S, Vogelaers D, Vandijck DM. What determines health-related quality of life among people living with HIV: An updated review of the literature. Arch Public Heal 2014; 72. DOI:10.1186/2049-3258-72-40.

29 Douab T, Marcellin F, Vilotitch A, et al. Health-related quality of life of people living with HIV followed up in hospitals in France: Comparing trends and correlates between 2003 and 2011 (ANRS-VESPA and VESPA2 national surveys). AIDS Care - Psychol Socio-Medical Asp AIDS/HIV 2014; 26: 29-40.

30 Monteiro F, Canavarro MC, Pereira M. Factors associated with quality of life in middle-aged and older patients living with HIV. AIDS Care 2016; 28: 92-8.

31 Herrmann S, McKinnon E, Hyland NB, et al. HIV-related stigma and physical symptoms have a persistent influence on health-related quality of life in Australians with HIV infection. Health Qual Life Outcomes 2013; 11. 
DOI:10.1186/1477-7525-11-56.

32 Engelhard EAN, Smit C, Van Dijk PR, et al. Health-related quality of life of people with HIV: An assessment of patient related factors and comparison with other chronic diseases. AIDS 2018; 32: 103-12.

33 Bakas T, McLennon SM, Carpenter JS, et al. Systematic review of healthrelated quality of life models. Health Qual. Life Outcomes. 2012; 10. DOI:10.1186/1477-7525-10-134.

34 Home - HIV Outcomes. https://hivoutcomes.eu/ (accessed July 21, 2020). 



\section{Figures}

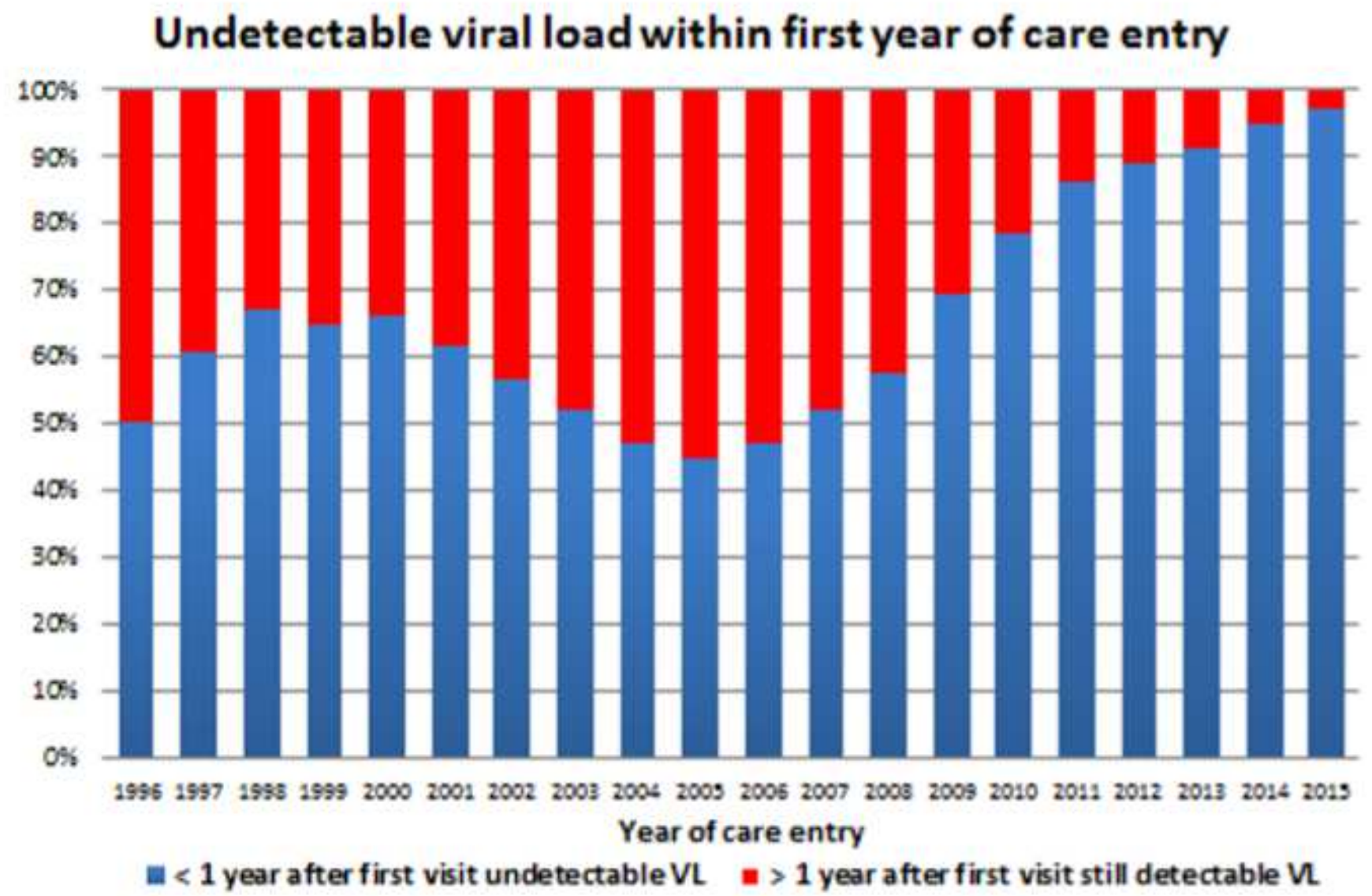

Figure 1

Starting in 2005, an increasing number of patients started HIV therapy soon after care entry. By 2015 , $96 \%$ of patients had full viral control in the first year of HIV care (indicator 1, figure 1). 


\section{Mortality within the first year of care entry and overall mortality}

$5,00 \%$

$4,50 \%$

$4,00 \%$

$3,50 \%$

$3,00 \%$

$2,50 \%$

$2,00 \%$

$1,50 \%$

$1,00 \%$

$0,50 \%$

$0,00 \%$

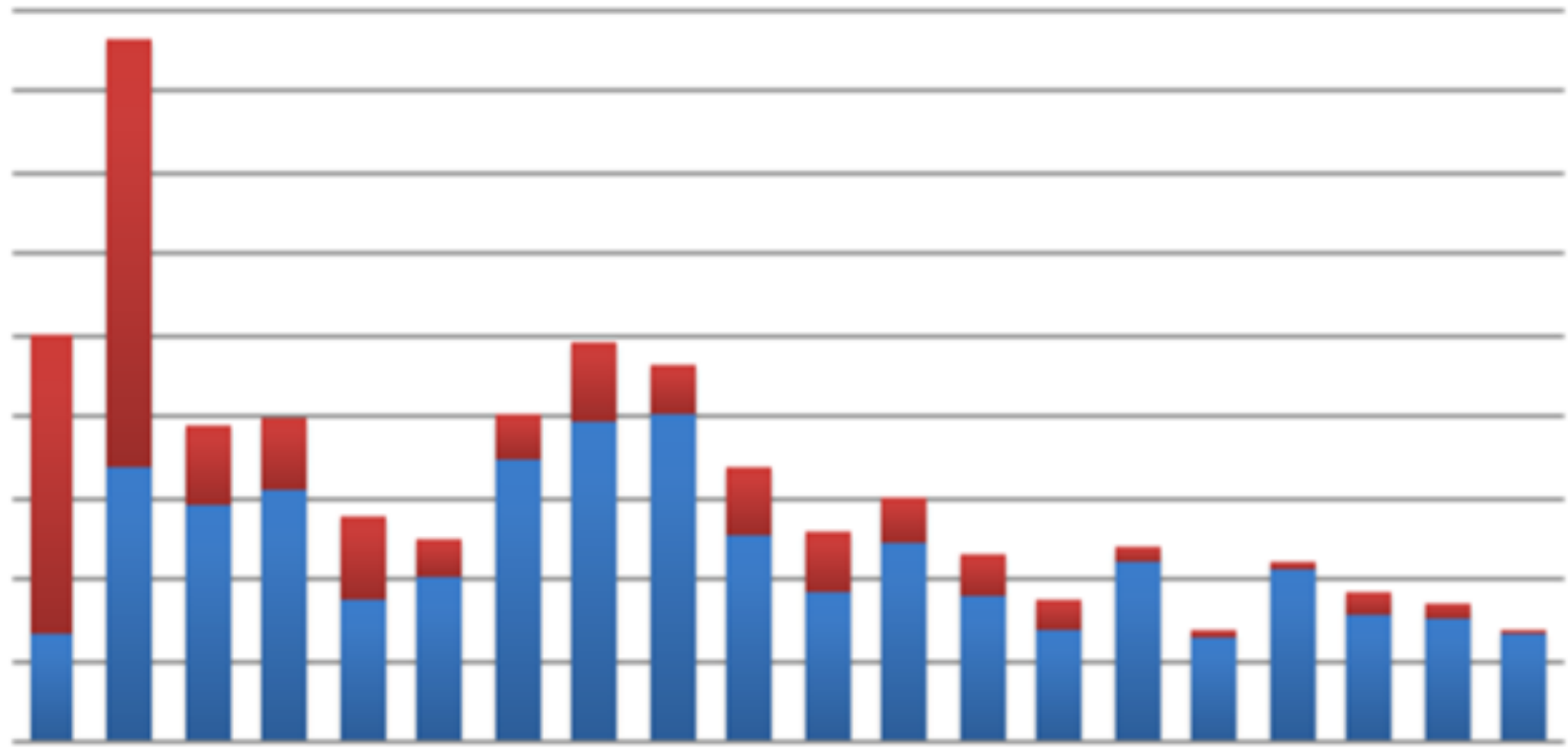

19961997199819992000200120022003200420092006200720082009201020112012201320142019 Year of death

$\|$ Deceosed $>1$ year ofter fir $z$ visit $\quad$ Deces sed $<1$ year o fter fir $z$ visit

Figure 2

Mortality rates in the first year of care were very low and were also very stable over time $(<1 \%)$ (indicators 3 and 9 ; figure 2). 


\section{Retention in care 2015}

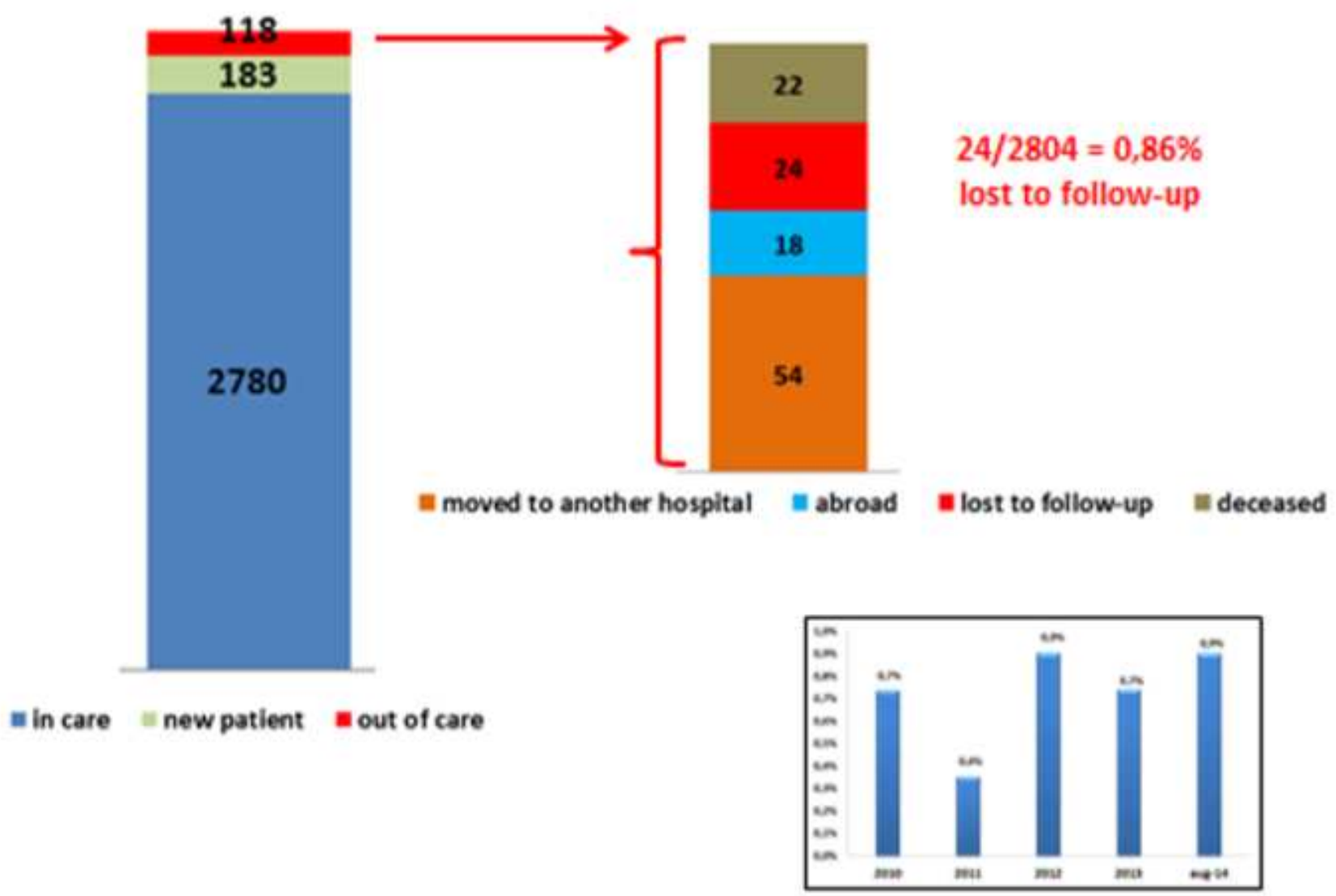

Figure 3

With $0.86 \%$ of patients lost to follow up (24 of the 2780 patients), the percentage of patients retained in care in 2015 was $99,14 \%$ (indicator 4 ; figure 3 ). 


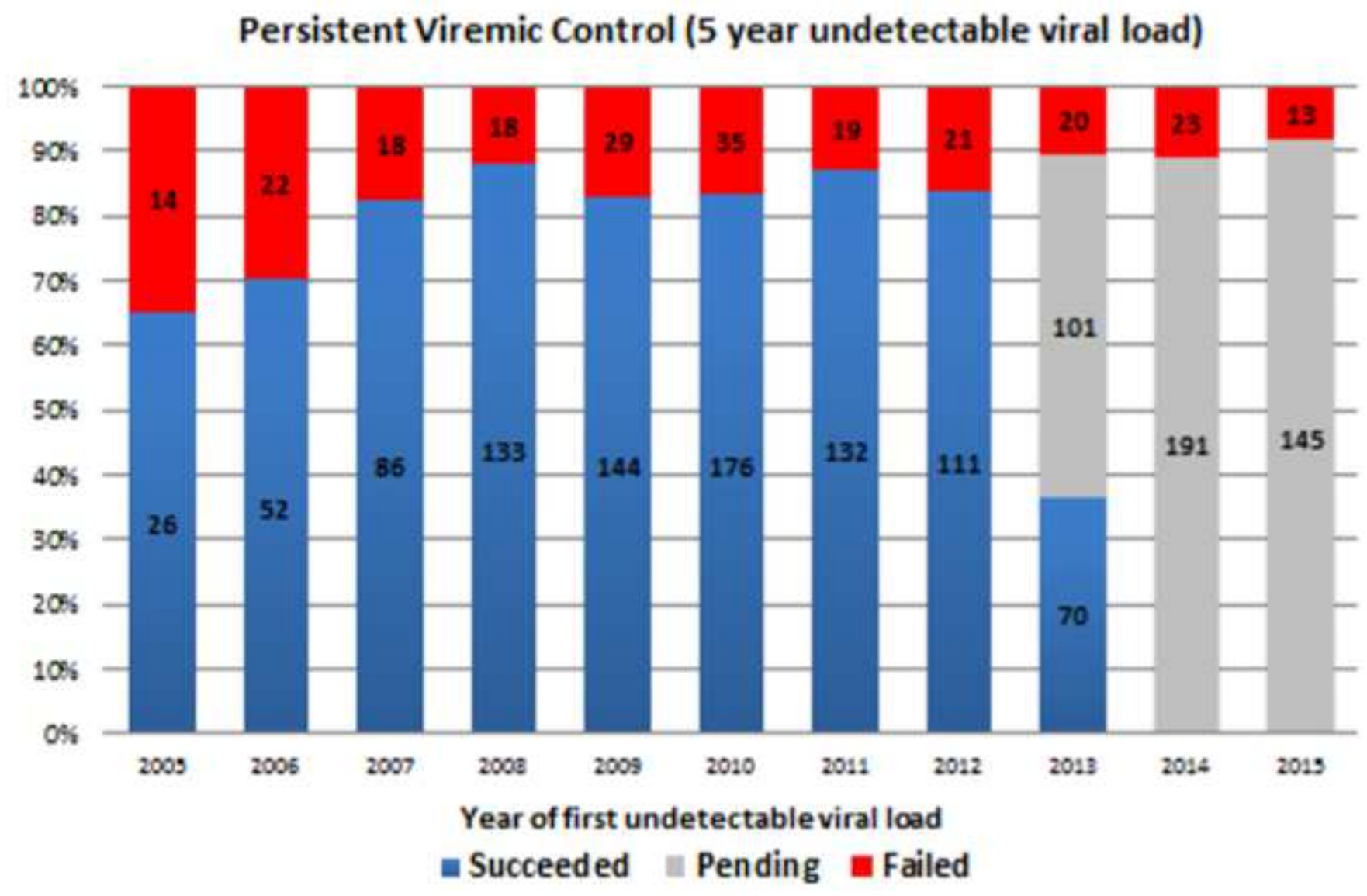

Figure 4

Persistence of viral control (for 5 years) increased from $65 \%$ of patients with full viral suppression in 2003 to a stable $90 \%$ in more recent years (indicator 5; figure 4). 


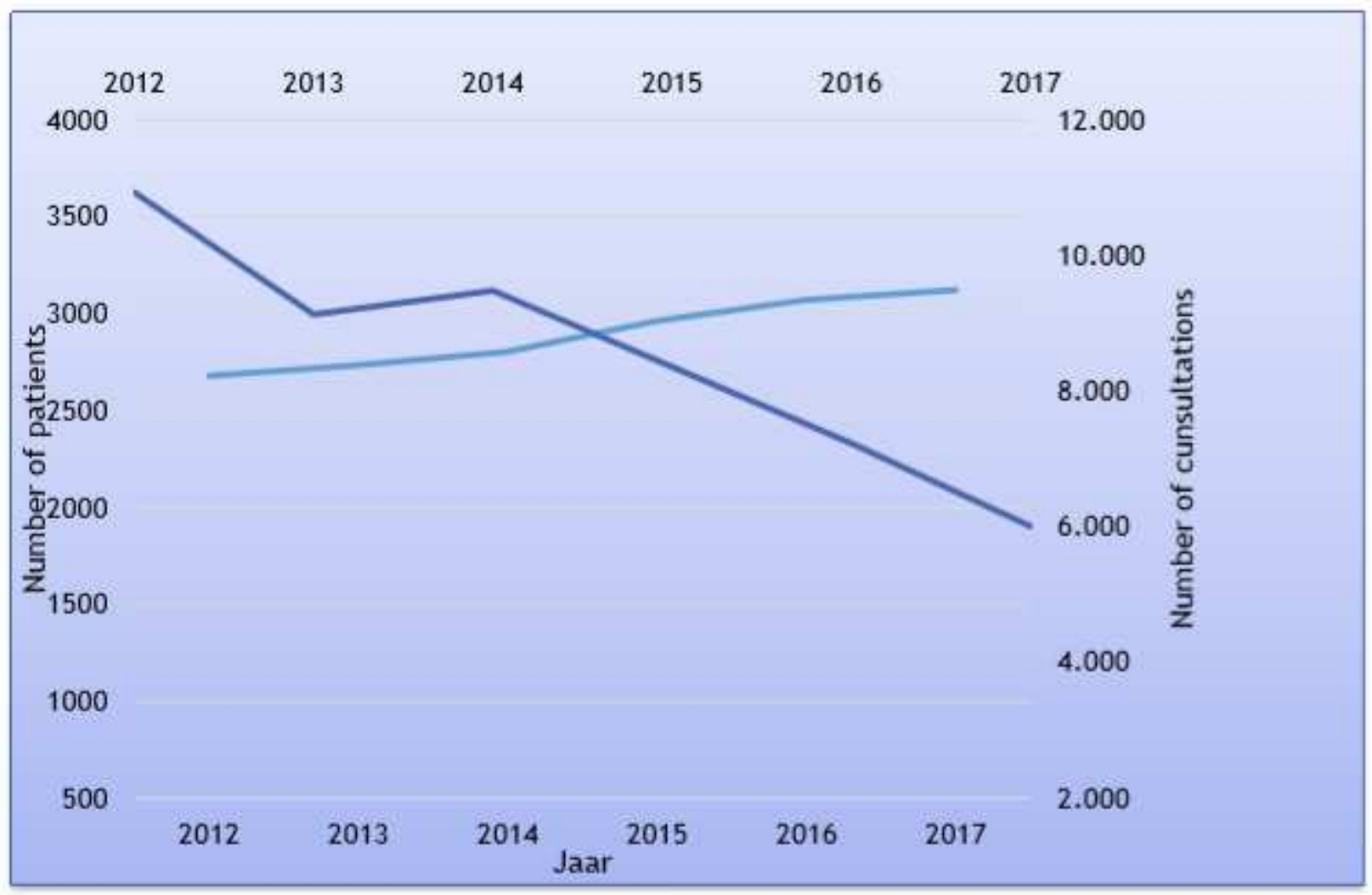

\section{Figure 5}

a decline in total laboratory costs and outpatient department (OPD) consultation costs despite growth in the patient population.

\section{Supplementary Files}

This is a list of supplementary files associated with this preprint. Click to download.

- table1.pdf

- Table2.pdf

- AppendixA.pdf 